\title{
An orthotropic damage model for crash simulation of composites
}

\author{
W. Wang ${ }^{1}$, F. H. M. Swartjes ${ }^{1} \&$ M. D. Gan ${ }^{2}$ \\ ${ }^{1} B U$ Automotive \\ ${ }^{2}$ Centre of Lightweight Structures TUD-TNO, \\ TNO Science and Industry, Delft, The Netherlands
}

\begin{abstract}
In this paper, a practical orthotropic damage model is developed and tested for composite materials during crash. The model uses the Hashin's failure criteria in which the fibre and matrix failures are described explicitly, both in tension and compression. A linear softening degradation is proposed and a close-form solution of the corresponding damage parameter is provided.

To reduce the mesh dependency, an embedded discontinuous element is proposed. It is a virtual embedded element in the sense that an actual element is divided into two zones, one elastic (undamaged) zone and one localization (damaged) zone. Their equivalence is preserved by constraining the kinematics and equilibrium equations. Since the damage zone is introduced into the element, the corresponding dissipated energy due to damage is fixed, independent of the element size.

The numerical simulations using the developed damage model show a mesh objective result and correlate well with the energy dissipation in dynamic 3-point bending experiments. It is concluded that an accurate material calibration is crucial for the success of failure simulations. The traditional strain mapping cannot be applied to failure problems in which the strain distribution is highly non-uniform. Material parameter calibration should take into account the ratio of the failure zone and the gauge length. A practical approximation formula is provided.
\end{abstract}

Keywords: composite, damage model, crash simulation, embedded element.

\section{Introduction}

The crash simulation of composites is particularly difficult due to the complexity of its physics. First, the failure process involves multiscales and is difficult to 
observe experimentally. Second, the softening effect leads to the well known numerical simulation difficulties, cf. mesh dependency, instability and bifurcation.

In this paper, a practical orthotropic damage model is developed and tested for composite materials during crash. This new model is implemented in LSDYNA as a user-defined material model. The related issues such as the mesh dependency and material calibrations of the corresponding damage parameters are addressed.

\section{Continuum damage constitutive relationship}

For plane stress situations in the continuum damage approach, the effective stress $\hat{\boldsymbol{\sigma}}$ is related to the total strain $\boldsymbol{\varepsilon}$ by the elastic stiffness tensor $\mathbf{C}_{0}$

$$
\hat{\boldsymbol{\sigma}}=\mathbf{C}_{0} \boldsymbol{\varepsilon}
$$

The effective stress is mapped to the true stress by the damage operator $\mathbf{M}$

$$
\boldsymbol{\sigma}=\mathbf{M} \hat{\boldsymbol{\sigma}}, \quad \mathbf{M}=\left[\begin{array}{ccc}
1-D_{1} & 0 & 0 \\
0 & 1-D_{2} & 0 \\
0 & 0 & 1-D_{12}
\end{array}\right]
$$

where $\mathrm{D}_{1}, \mathrm{D}_{2}$ and $\mathrm{D}_{12}$ are the damage parameters. Inserting Equation (1) into Equation (2) yields

$$
\boldsymbol{\sigma}=\mathbf{M C}_{0} \boldsymbol{\varepsilon}=\mathbf{C} \boldsymbol{\varepsilon}
$$

in which the damaged stiffness tensor $\mathbf{C}$ reads

$$
\begin{gathered}
\mathbf{C}=\frac{1}{\phi}\left[\begin{array}{ccc}
\left(1-D_{1}\right) E_{11} & \left(1-D_{1}\right) v_{21} E_{22} & 0 \\
\left(1-D_{2}\right) v_{12} E_{11} & \left(1-D_{2}\right) E_{22} & 0 \\
0 & 0 & \left(1-D_{12}\right) \phi G
\end{array}\right] \\
\phi=1-v_{12} v_{21}
\end{gathered}
$$

with $\mathrm{E}_{11}, \mathrm{E}_{22}, \mathrm{G}, v_{12}$ and $v_{21}$ are the elasticity parameters of the undamaged lamina. Note that when damage occurs $\left(D_{i}>0\right)$, the stiffness tensor is not symmetric. To preserve a symmetric stiffness tensor, the Poisson's ratio can be related to the damage parameters [3]. 


\section{Hashin's failure criteria}

Hashin's criteria [2] refers to an unidirectional fibre reinforced composite with five failure modes:

1) Fibre tensile and compression modes

$$
e_{f}^{2}=\left(\frac{\sigma_{11}}{X}\right)^{2}-1\left\{\begin{array}{l}
\geq 0 \text { failed } \\
<0 \text { elastic }
\end{array}\right.
$$

2) Matrix tensile and compression modes

$$
e_{m}^{2}=\left(\frac{\sigma_{22}}{Y}\right)^{2}-1\left\{\begin{array}{l}
\geq 0 \text { failed } \\
<0 \text { elastic }
\end{array}\right.
$$

3) Shear mode

$$
e_{s}^{2}=\left[\frac{\tau}{S}\right]^{2}-1 \begin{cases}\geq 0 & \text { failed } \\ <0 & \text { elastic }\end{cases}
$$

where $X$ and $Y$ are fiber and matrix strengths and $S$ is the shear strength. Note that the compression and tensile modes are treated in the same way. For notation simplicity, the sub indexes for the tensile and compression strength parameters are omitted.

Note that Hashin's criteria have a complete decoupling of the fibre and matrix failure modes from shear failure. Therefore, the failure criteria may overestimate the strength of a lamina when shear is present. However, it should be mentioned that measuring the softening part of the stress-strain curve is very difficult even in a uniaxial test condition. A multi-axial loading surface is almost impossible to be validated. Furthermore, the damage evolution or degradation law is, in most cases, in an uncoupled fashion. Therefore, it is convenient to treat all failure criteria to be independent.

It must be emphasised that although the failure modes and the degradation law (which will be introduced in the next section) are decoupled, the complete stress-strain relationship still contains a coupling due to the stiffness matrix $\mathbf{C}$ (see Equation 3).

\section{Degradation rule}

Damage occurs when one of the Hashin's criteria is satisfied. For simplicity, we assume a linear degradation law (see Figure 1) after the initial strength $\sigma_{0}$ is exceeded. Consequently, the damage parameter D can be determined by the following relationship

$$
\sigma=E(D) \varepsilon=E_{0}(1-D) \varepsilon=\sigma_{0}-h\left(\varepsilon-\varepsilon_{0}\right)
$$


where $E_{0}$ is the elastic modulus, $\varepsilon_{0}=\sigma_{0} / E_{0}$ the initial failure strain and $h$ is the softening parameter, respectively.

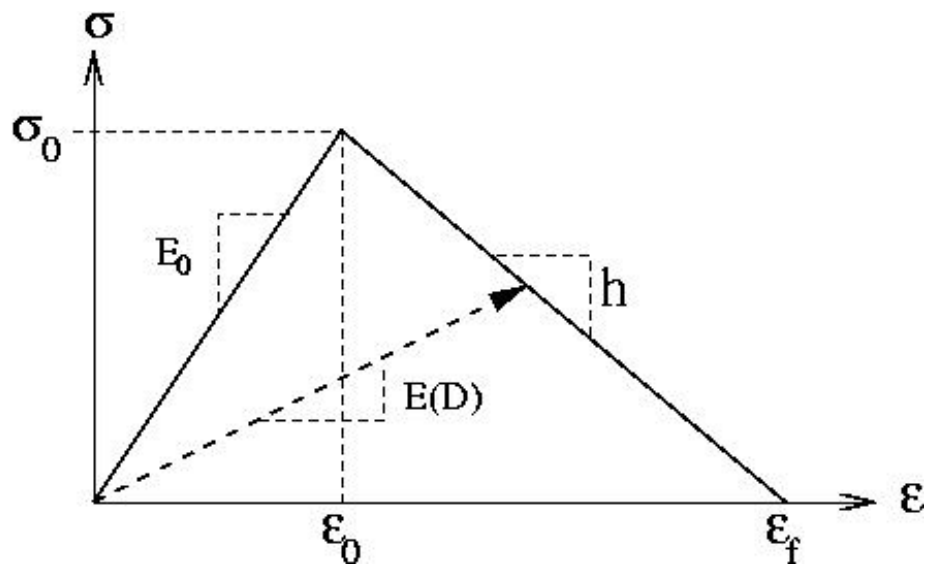

Figure 1: A linear stiffness degradation.

It must be emphasised that Equation (9) holds for materials in the localisation zone. Two issues related to this localisation problem should be addressed:

1) The material calibration of localisation related parameters such as the softening parameter $\mathrm{h}$ and the final failure strain $\varepsilon_{f}$. Note that the strain field is not uniform as soon as localisation occurs. Therefore, the standard strain mapping in uniaxial tensile examples: $\varepsilon=u / L$ where $L$ is the gauge length and $u$ the deformation, is not valid. The results of the experiments should be interpreted carefully.

2) The mesh dependent problem. For a tensile bar with a uniform cross section area A, only one element will follow the failure path. Consequently, the total dissipation energy of the tensile bar reads

$$
W_{d i s s}=\frac{1}{2} \sigma_{0}\left(\varepsilon_{f}-\varepsilon_{0}\right) l \mathrm{~A}
$$

where 1 is the length of the failure element. Clearly, the energy dissipation is dependent on the element size and, therefore, the simulation result is mesh dependent.

These two issues will be discussed in the next section. First, the mesh dependence problem is solved by introducing a localisation zone into a standard finite element. Next, the corresponding localisation related parameters are investigated and their relationships are outlined. Finally, formula will be provided for practical approximation of the final failure strain and the softening parameter. 


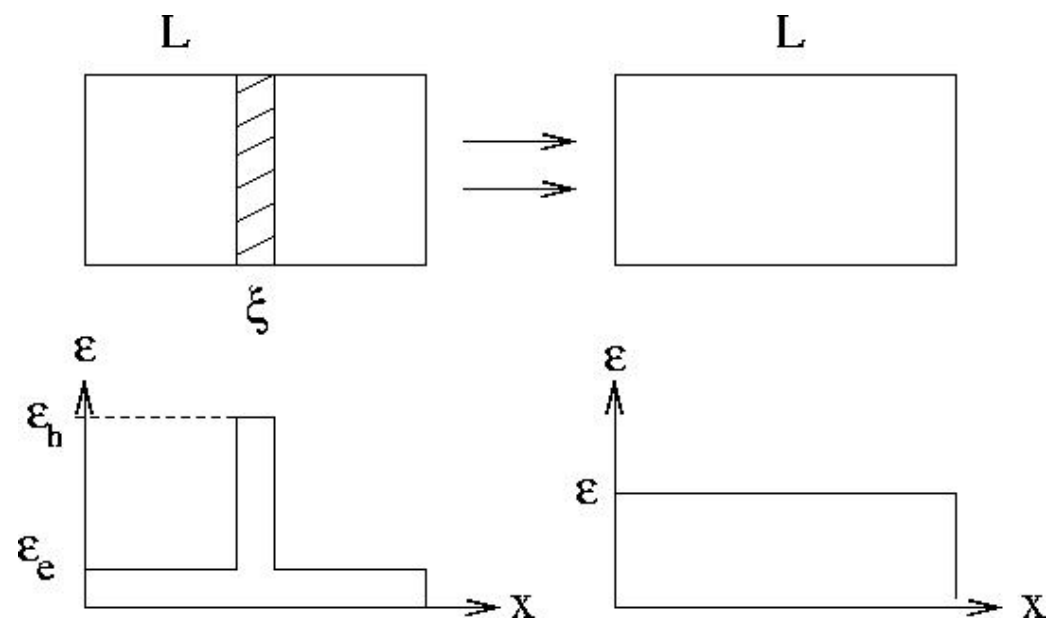

Figure 2: Embedded localisation element (left) and its equivalent element (right).

\section{Embedded localization element}

To reduce the mesh dependency, an embedded discontinuous element is proposed. It is a virtual embedded element in the sense that an actual element is divided into two zones, one elastic (undamaged) zone and one localization (damaged) zone. Their equivalence is preserved by constraining the kinematics and equilibrium equations. Since the damage zone is introduced into the element, the corresponding dissipated energy due to damage is fixed, independent of the element size. Therefore, mesh dependency with respect to dissipated energy can be removed.

Assume that the length of the damage zone is $\xi$ and the length of the element is $\mathrm{L}$, see Figure 2. When damage occurs, the strain localised in the damage zone is $\varepsilon_{\mathrm{h}}$ and the strain at the elastic zone is $\varepsilon_{\mathrm{e}}$. If we smear the localisation zone and make an equivalent element, the following kinematics and equilibrium equations must be satisfied

$$
\begin{aligned}
& \xi \varepsilon_{h}+(L-\xi) \varepsilon_{e}=L \varepsilon=u \\
& E_{0} \varepsilon_{e}=E_{h} \varepsilon_{h}=E_{L} \varepsilon=\sigma
\end{aligned}
$$

where $E_{0}$ is the elastic modulus, $E_{h}$ the stiffness at the damage zone and $E_{L}$ the equivalent (element) elastic modulus, respectively

$$
\begin{aligned}
& E_{h}=(1-d) E \\
& E_{L}=(1-D) E
\end{aligned}
$$


Note that $d$ and $D$ are damage parameters of the embedded localisation element and the equivalent element, respectively.

From Equations (11-14) the localisation strain $\varepsilon_{h}$ can be related to the equivalent (element) strain $\varepsilon$

$$
\varepsilon_{h}=\frac{\varepsilon}{1+d(r-1)}, \quad r=\frac{\xi}{L}
$$

and the local damage parameter $d$ can be evaluated and then transferred to the global (element) damage parameter

$$
D=\frac{r d}{1+d(r-1)}
$$

Note that the localisation strain $\varepsilon_{h}$ is a function of damage parameter $\mathrm{d}$. This local damage parameter can be determined by inserting Equations (13)-(15) into the linear softening law (9)

$$
d=\frac{(E+h)\left(\varepsilon-\varepsilon_{0}\right)}{E \varepsilon+(E+h) \varepsilon_{0}(r-1)}
$$

With a known element strain $\varepsilon$, the local damage parameter can be directly determined. This is the advantage of a linear softening assumption, which makes the stress update simple and straightforward.

Note that the softening parameters should be carefully calibrated. The traditional strain mapping cannot be applied to failure problems in which the strain distribution is highly non-uniform. Material parameter calibration should take into account the ratio of the failure zone and the gauge length.

For the final failure, $d=D=1$, from Equation (15), the local failure strain $\varepsilon_{f}$ and the element failure strain $\chi$ have relationship

$$
\varepsilon_{f}=\frac{\chi}{r} \quad r=\frac{\xi}{L}
$$

Suppose one uses one element to simulate a tensile bar with a gauge length $\mathrm{L}$. The material failure strain $\varepsilon_{\mathrm{f}}$ reads

$$
\varepsilon_{f}=\frac{\chi}{r}=\frac{L}{\xi} \frac{u}{L}=\frac{u}{\xi}
$$

where $\xi$ is the length of the damage zone. Note that the material failure strain is obtained by dividing the deformation by the length of the damage zone and not the gauge length. 


\section{Numerical simulations}

\subsection{Mesh sensitivity analysis}

A mesh sensitivity analysis is performed by simulating the fracture behaviour of the juvenile part of a dogbone specimen with a multi-element FE model. Three different meshes are used with element size of $2 \mathrm{~mm}, 1 \mathrm{~mm}$ and $0.5 \mathrm{~mm}$ for mesh 1, 2 and 3, respectively (see Figure 3).

The same material data are used for the LS-DYNA material model 58 and the new user-defined material model (with the assumption that the failure zone is equal to $0.1 \mathrm{~mm}$ ).

In Figure 4 the results of the mesh sensitivity analysis are plotted. Clearly, the global force is independent on the mesh size.
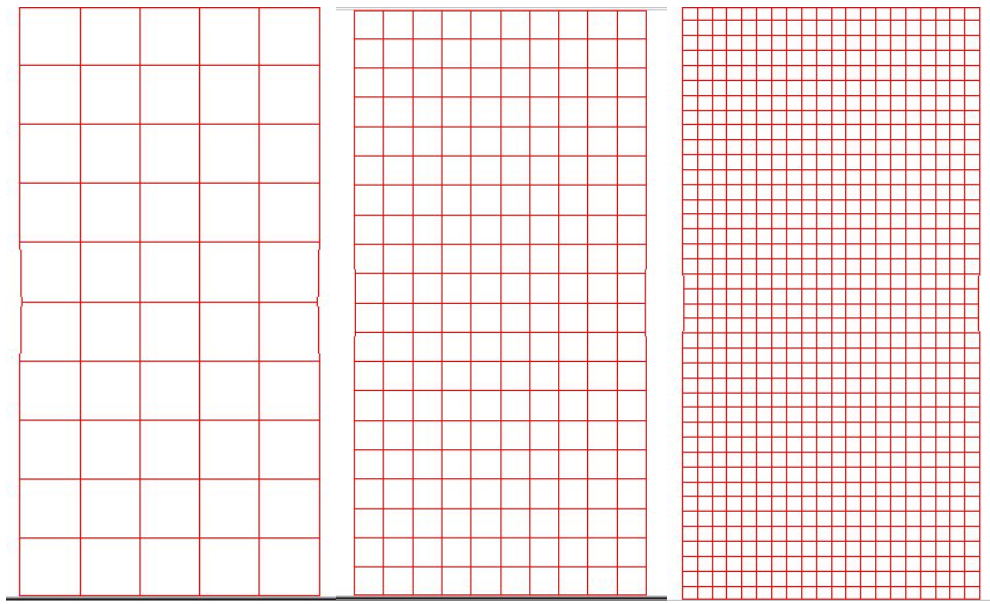

Figure 3: Mesh sensitivity analysis: three meshes with element size $2 \mathrm{~mm}$ (left), $1 \mathrm{~mm}$ (middle) and $0.5 \mathrm{~mm}$ (right).
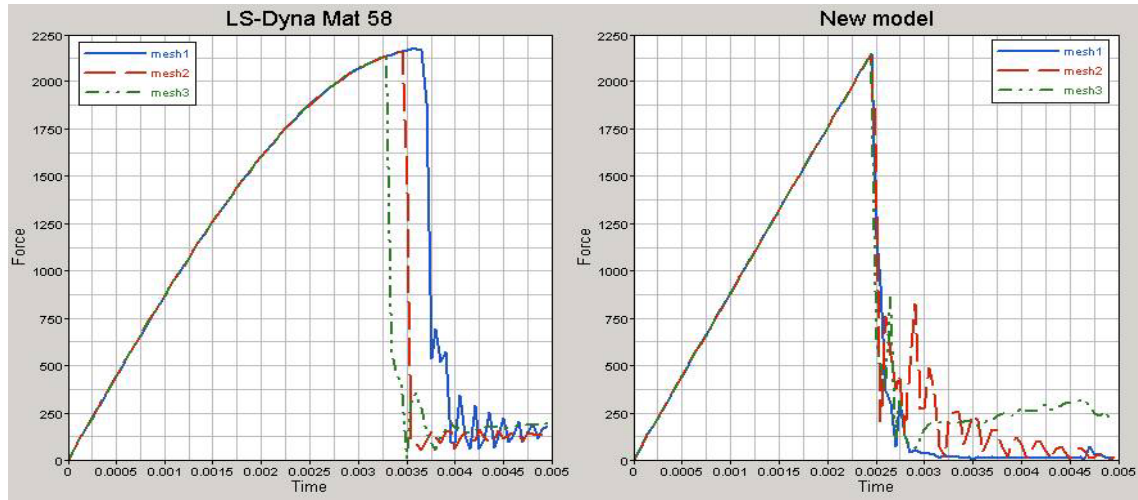

Figure 4: Mesh sensitivity analysis for the LS-DYNA material model 58 (left) and the user-defined model (right). 


\subsection{Three-point bending tests}

Next, numerical simulations on three-point bending experiments were performed using the new developed composite model. These US bumper standard experiments (NHTSA 49 CFR Part 581: Bumper Standard) were performed on U-shaped GMT bumper beams with an aluminum impactor having a total mass $778 \mathrm{~kg}$ and initial impact velocity of $2.30 \mathrm{~m} / \mathrm{s}$.

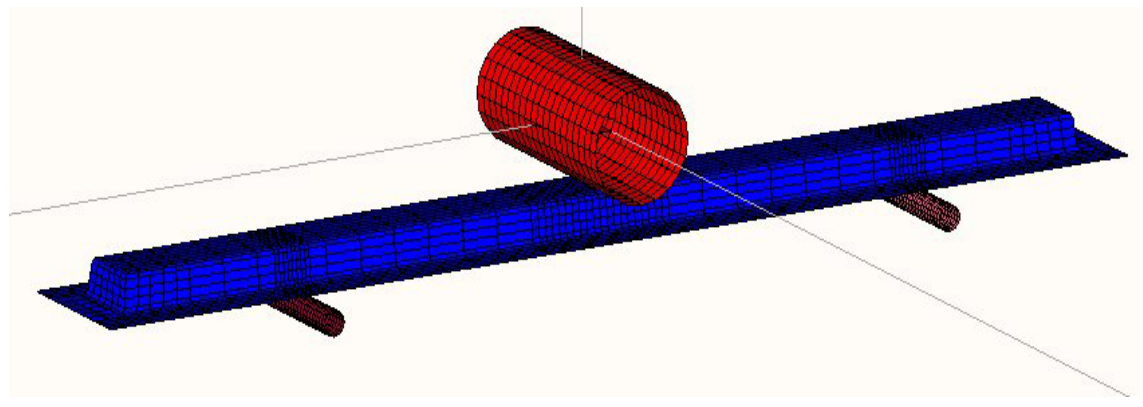

Figure 5: $\quad$ FE model for the three-point bending tests on composite U-beams.

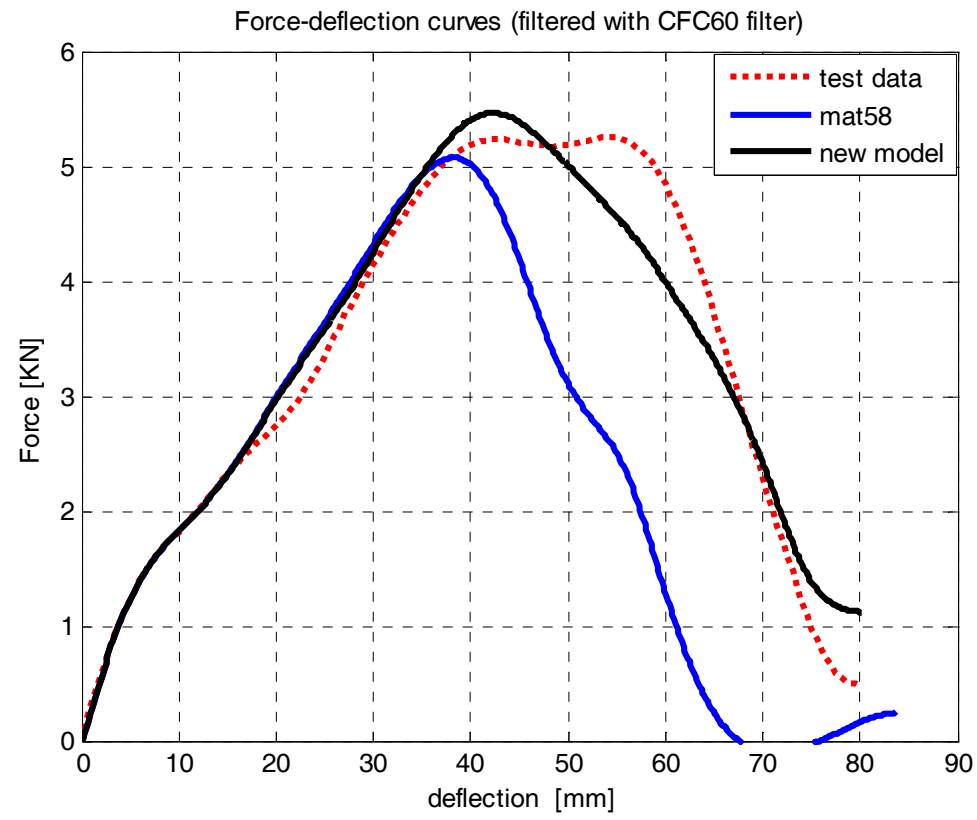

Figure 6: Force-deflection curves from test and simulation for the dynamic three-point bending experiments on composite U-beams. 
The FE model of the experimental set-up is plotted in Figure 5. The same material parameters are used for the LS-DYNA material model 58 and the new user-defined material model.

In Figure 6 the contact forces for the experiment and numerical simulations are plotted (all data are filtered with a CFC60 filter). Both models predict the shape of the force-deflection curve accurate until the onset of failure. It is observed that the new user-defined material model captures the softening range better than the original LS-DYNA material model 58.

\section{Conclusions}

An orthotropic damage model is developed and tested for composite materials. The model uses the Hashin's failure criteria in which the fibre and matrix failures are described explicitly, both in tension and compression. A linear softening degradation is proposed and a close-form solution of the corresponding damage parameter is provided. This new model is implemented in LS-DYNA as a user-defined material model.

To reduce the mesh dependency, an embedded discontinuous element is proposed. It is a virtual embedded element in the sense that an actual element is divided into two zones, one elastic (undamaged) zone and one localisation (damaged) zone. Their equivalence is preserved by constraining the kinematics and equilibrium equations. Since the damage zone is introduced into the element, the corresponding dissipated energy due to damage is fixed, independent on the element size. Therefore, mesh dependency with respect to the dissipated energy is removed.

It is concluded that an accurate material calibration is crucial for successful failure simulations. The traditional strain mapping cannot be applied to failure problems in which the strain distribution is highly non-uniform. Material parameter calibration should take into account the ratio of the failure zone and the gauge length. A practical approximation formula is provided.

\section{Reference}

[1] Haan, P.A.J. de, Swartjes, F.H.M and Wang, W. "Failure of composite material under impact loading- WP4: Numerical simulation of three-point bending experiments on bumper beams", TNO Science and Industry, Internal report 04.OR.SA.032.1/FSW, 2004.

[2] Hashin Z. "Failure criteria for unidirectional fiber composites", Composite struct. 32, 255-264 (1980).

[3] Schweizerhof, K., Weimar, K., Münz, Th. and Rottner, Th. "Crashworthiness analysis with enhanced composite material models in LS-DYNA -Merits and limits", $5^{\text {th }}$ Ls-Dyna User's Conference, Southfield, Michigan, USA (1998). 